\title{
Right Ventricle Foreign Body: A Case of a Migrating Iliac Stent Entrapped in the Tricuspid Valve
}

\author{
Majd Ibrahima, b, Auroa Badina, Sudhir Mungee $^{a}$
}

\begin{abstract}
We report a 27-year-old man with a history of atrial fibrillation, pericarditis, and May-Thurner syndrome who underwent a venoplasty with two self-expandable stents in the left common iliac vein. One year later, he presented with tricuspid valve insufficiency and a dilated right atrium. His echocardiogram showed a foreign body in the right ventricle enmeshed in the tricuspid valve. This was identified as a migrating iliac stent confirmed by the absence of one of the peripheral stents on venogram. He failed a percutaneous approach to retrieve the stent and underwent subsequent surgical extraction with open sternotomy. There was significant damage to the tricuspid valve necessitating valvuloplasty and annuloplasty. We believe the migrated stent may have been relatively small for this patient's vascular anatomy and this serious complication could have been avoided by proper stent size selection. Fortunately, our patient is now asymptomatic with no functional limitations.
\end{abstract}

Keywords: Iliac vein migrating stent; Tricuspid regurgitation; Enmeshed stent

\section{Introduction}

Dislodgement and migration of venous stents into the right ventricle are rare but serious complications. The incidence of stent migration as a complication of endovascular stenting is reported to be as low as 3\% of all percutaneous stenting procedures. Our case highlights this rare and serious complication of endovascular therapy and stresses the importance of choosing appropriate stent size and deployment techniques to minimize

\section{Manuscript accepted for publication November 14, 2014}

aDepartment of Internal Medicine, Division of Cardiology, University of Illinois College of Medicine at Peoria, OSF Saint Francis Medical Center, Peoria, IL, USA

${ }^{b}$ Corresponding Author: Majd Ibrahim, Department of Internal Medicine, Division of Cardiology, University of Illinois College of Medicine at Peoria, OSF Saint Francis Medical Center, 530 NE Glen Oak Ave., Peoria, IL 61637, USA. Email: ibrahimm@uic.edu

doi: http://dx.doi.org/10.14740/jmc1991w the risk of stent migration.

\section{Case Report}

A 27-year-old man with a history of atrial fibrillation and remote pericarditis presented to an outside hospital with recurrent left lower extremity edema. His physical exam and initial venous studies of the left lower extremity suggested proximal obstruction. Further investigations included venography and intravascular ultrasound of the left iliac vein and inferior vena cava (IVC). These imaging studies revealed an area of compression from the adjacent right iliac artery causing narrowing of the lumen to $9 \mathrm{~mm}$. This was deemed hemodynamically significant and correlated with the patient's symptoms. Two self-expandable $14 \times 40 \mathrm{~mm}$ Bard Luminexx stents (C.R. Bard, Murray Hill, NJ, USA) were deployed in the left common iliac vein at the area of stenosis. During the deployment of the second stent, the stent migrated into the IVC. It stayed on the guidewire and was recaptured with the balloon and repositioned in the desired location proximal to the first stent. Following deployment, an angiogram showed the two stents overlapping with the second proximal stent covering the targeted stenosis. His post-operative course was complicated by moderate bilateral pneumothorax which resolved after chest tube placement.

One year after venoplasty, the patient presented to our emergency department with new onset chest pain, dyspnea on exertion, ascites, scrotal, and bilateral lower extremity edema. A trans-thoracic echocardiogram revealed a bright foreign body which appeared to be a stent in the right ventricle immediately adjacent to the tricuspid valve causing moderate tricuspid insufficiency and a dilated right atrium (Fig. 1A, B, C) (Supplementary videos, www.journalmc.org). Venography of the common and left iliac veins demonstrated a protrusion of one iliac stent into the patient's IVC and the absence of the other stent (Fig. 2). Therefore, a migrated iliac vein stent was presumed to be the source of this foreign body. A Bard IVC filter was placed to prevent mobilization of the remaining iliac stent to the heart. A percutaneous endovascular approach with a $10 \mathrm{~mm}$ straight snare was initially attempted to retrieve the migrated stent. The stent's position near the tricuspid valve was concerning for papillary muscle and chordae tendineae damage and therefore the percutaneous attempt was aborted. The 

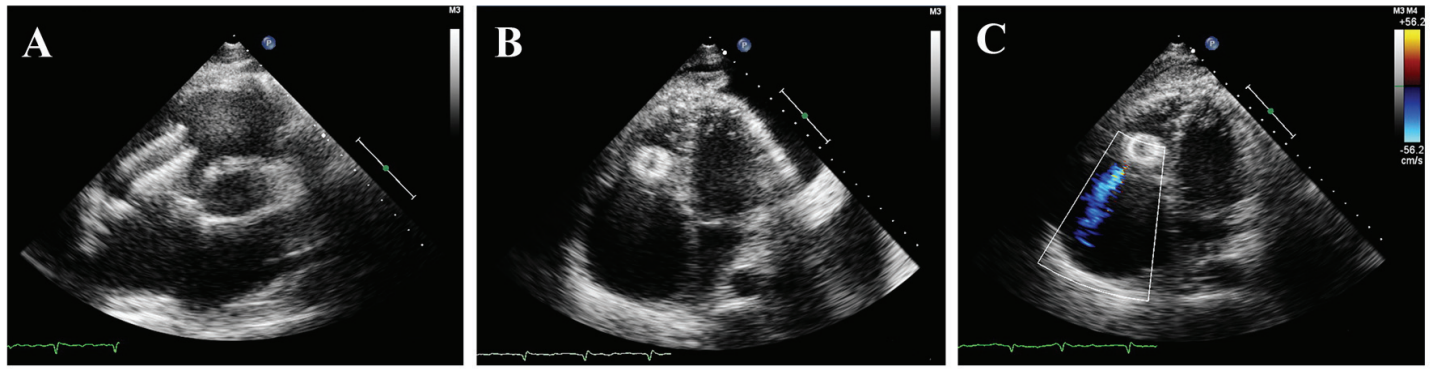

Figure 1. Short-axis view (A) and apical four view (B) images of the heart by transthoracic echocardiography, showing migrated iliac vein stent in the right ventricle enmeshed in the tricuspid valve, causing moderate tricuspid regurgitation by color Doppler (C).

patient was referred for an operative extraction and underwent median sternotomy with cardiopulmonary bypass. The stent which was adherent to the anterior and posterior leaflets of the tricuspid valve was removed and the patient required tricuspid valvuloplasty, as well as annuloplasty due to a densely scarred valve using a Carpentier-Edwards annuloplasty ring (Edwards Life-sciences, Irvine, CA, USA). Post-operative echocardiogram demonstrated no tricuspid regurgitation. The IVC filter was left in place as a preventative measure.

Two years later, the patient developed constrictive pericarditis with heart failure and eventually underwent pericardial stripping and surgical MAZE procedure for concurrent atrial fibrillation. At his recent clinic follow-up, he reported no functional limitation and was able to regularly exercise. His echocardiography demonstrated trace-mild tricuspid regurgitation and normal ejection fraction.

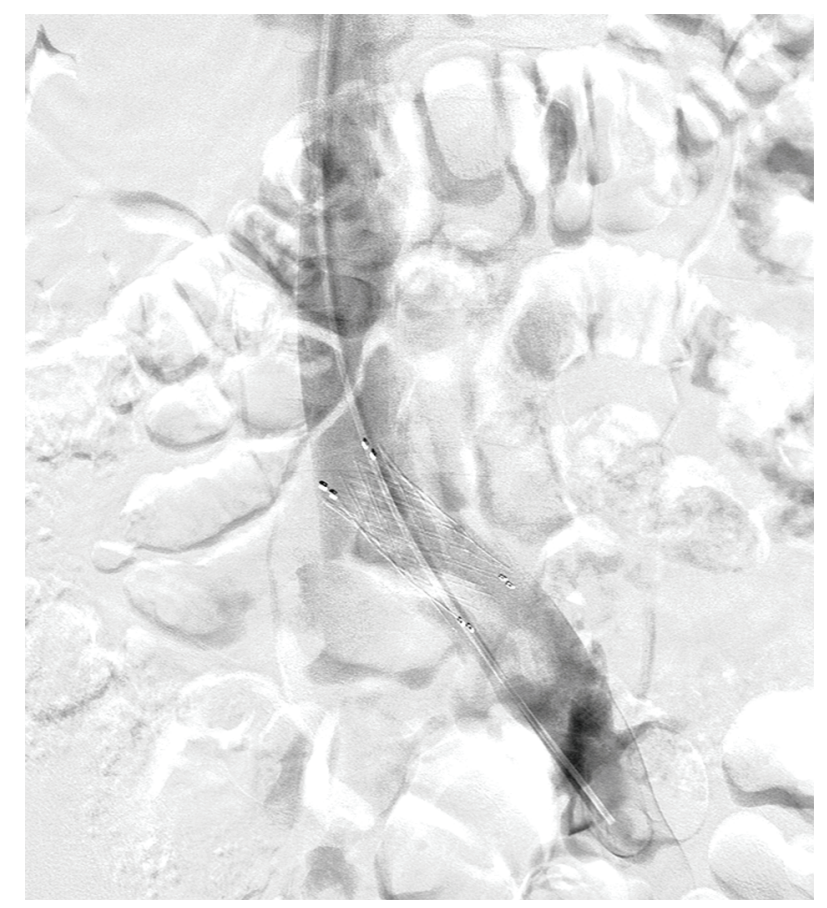

Figure 2. Venography of the common and left iliac veins demonstrating protrusion of one left iliac stent into the IVC.

\section{Discussion}

The chronic compression of the left iliac vein by the adjacent right common iliac artery and lumbosacral spine may cause pain, edema or deep venous thrombosis. This condition is known as iliac vein compression syndrome or May-Thurner syndrome and has been associated with unprovoked left iliofemoral deep venous thrombosis and chronic venous insufficiency [1]. Hemodynamically significant lesions are indicated for intervention. Balloon dilation and stenting have been found to be superior to conventional surgical treatment and should be considered the first line of therapy for patients suffering from chronic iliocaval venous obstruction [2].

Dislodgement and migration of venous stents into the right ventricle are rare but serious complications. The incidence of stent migration as a complication of endovascular stenting is reported to be as low as 3\% of all percutaneous stenting procedures [3]. Location of the lesion, deployment technique, appropriate stent size and external trauma are essential contributing factors to stent migration. Selection of the correct stent size is a major determinant of the procedure success. The diameter of the stent must be greater than that of the designed vein to prevent stent migration and compensate for neointimal growth [4].

Our case demonstrates a serious complication of endovascular therapy that could have been minimized by proper stent selection and deployment. The migrated stent may have been relatively small for this patient's vascular anatomy. Endovascular retrieval by snaring the stent is usually recommended before surgical approach. Unfortunately, this was potentially hazardous due to the size of the self-expandable Luminexx stent, its position relative to the tricuspid valve, and the substantial scarring of the valve. Thus, open surgical extraction had to be performed to safely remove the stent.

\section{Disclosure}

There is no source of support or grants for this manuscript, nor potential conflict of interest for any of the authors.

\section{Author Contributions}

All authors have participated in the intellectual content and re- 
viewed the final version of this manuscript and approved it for publication.

\section{References}

1. May R, Thurner J. The cause of the predominantly sinistral occurrence of thrombosis of the pelvic veins. Angiology. 1957;8(5):419-427.

2. Mussa FF, Peden EK, Zhou W, Lin PH, Lumsden AB,
Bush RL. Iliac vein stenting for chronic venous insufficiency. Tex Heart Inst J. 2007;34(1):60-66.

3. Bani-Hani S, Showkat A, Wall BM, Das P, Huang L, Al-Absi AI. Endovascular stent migration to the right ventricle causing myocardial injury. Semin Dial. 2012;25(5):562-564.

4. El Feghaly M, Soula P, Rousseau H, Chaiban F, Otal P, Joffre F, Cerene A. Endovascular retrieval of two migrated venous stents by means of balloon catheters. J Vasc Surg. 1998;28(3):541-546. 